\title{
FIXED SET SYSTEMS OF EQUIVARIANT INFINITE LOOP SPACES
}

\author{
STEVEN R. COSTENOBLE AND STEFAN WANER
}

\begin{abstract}
We develop machinery enabling us to show that suitable $G$-spaces, including the equivariant version of $B F$, are equivariant infinite loop spaces. This involves a "recognition principle" for systems of spaces which behave formally like the system of fixed sets of a $G$-space; that is, we give a necessary and sufficient condition for such a system to be equivalent to the fixed set system of an equivariant infinite loop space. The advantage of using the language of fixed set systems is that one can frequently replace the system of fixed sets of an actual $G$-space by an equivalent formal system which is considerably simpler, and which admits the requisite geometry necessary for delooping. We also apply this machinery to construct equivariant Eilenberg-Mac Lane spaces corresponding to Mackey functors.
\end{abstract}

\section{INTRODUCTION AND STATEMENT OF RESULTS}

If $G$ is a transformation group, the $G$-spaces which play the role of classifying spaces are frequently extremely complicated objects. For example, the equivariant version of the classifying space $B F$ of stable spherical fibrations must combine information about all the possible orthogonal representations of subgroups (to reflect the various linear actions of subgroups on fibers), as well as information about the monoids of equivariant homotopy equivalences associated with each such representation. As a consequence, equivariant classifying spaces must usually be constructed using bar construction-type machinery applied to complicated categories (see, e.g., [W2, W3]); hence their geometry is obscure. It is our goal here to describe machinery that can be used to prove that many classifying $G$-spaces, including equivariant $B F$, are equivariant infinite loop spaces, and hence the zeroth spaces of equivariant spectra [LMS]. Throughout, we take $G$ to be a finite group.

There are known "recognition principles" for nonequivariant infinite loop spaces; these are results that characterize infinite loop spaces, and take the form of a simple test enabling one to determine whether a given space admits deloopings of all orders. In the language of May [M1, M3], one such test consists of the determination of whether the candidate space admits an action by an $E_{\infty}$ operad. Equivariantly, an analogous recognition principle has been developed by Hauschild, May, and one of the present authors, and will be outlined here

Received by the editors January 25, 1989 and, in revised form, July 1, 1989.

1980 Mathematics Subject Classification (1985 Revision). Primary 54H15, 55D35, 55D40, $55 \mathrm{~F} 35$ 
(see Theorem 1(a) below). This principle can be used to show that suitable $G$-spaces are equivariant infinite loop spaces. However, this form of the recognition principle is not quite adequate to give deloopings of $G$-spaces such as $B F$, nor does it provide models for equivariant Eilenberg-Mac Lane spaces associated with ordinary $R O(G)$-graded cohomology with coefficients in a general Mackey functor [LMM].

The problem with an equivariant version of the recognition principle is twofold. Firstly, in view of the complexity of classifying $G$-spaces, it is difficult to construct models for these spaces that easily admit actions by equivariant $E_{\infty}$ operads. Equivariant $B F$ is a particularly difficult example. Secondly, the object being delooped may not be a $G$-space, but rather a functor on the orbit category, such as a Mackey functor. In order to address these problems, we shall show here that it is possible to extend the equivariant delooping machinery to cover systems of spaces which behave formally like systems of fixed sets of $G$-spaces [E]. This may be formalized as follows. Let $\mathscr{G}$ be the category whose objects are the spaces $G / H$ for $H \subset G$, and whose morphisms are the $G$-maps. A (based) $\mathscr{G}$-space is a contravariant functor from $\mathscr{G}$ to the category of (based) topological spaces. Given a based $\mathscr{G}$-space $\mathscr{X}$, we give a criterion, in terms of $\mathscr{X}$, for the existence of an equivariant infinite loop space $L(\mathscr{X})$ such that the system of fixed-sets $L(\mathscr{X})^{H}$ is equivalent to $\mathscr{X}$ as a $\mathscr{G}$-space.

As a consequence of this theory, we deduce an (embarrassingly long overdue) proof that equivariant $B F$ has the $G$-homotopy type of an equivariant infinite loop space. We also construct explicit models for the equivariant Eilenberg-Mac Lane spaces associated with Mackey functors. The theory presented here will eventually form part of a more comprehensive treatment of equivariant infinite loop space theory [CHMW] (although work on this is not expected to begin for some time).

Now let us introduce some notation and state things more precisely. Let $\mathscr{U}$ be a complete G-universe: an infinite-dimensional orthogonal real representation of $G$ which contains infinitely many copies of each representation (including the trivial one). For $V \subset \mathscr{U}$ finite dimensional and $G$-invariant, denote the one-point compactification of $V$ by $S^{V}$. If $X$ is a $G$-space with a stationary basepoint, then the loop space $\Omega^{V} X$ of based maps from $S^{V}$ to $X$ is acted on by $G$ via conjugation. If we denote $X \wedge S^{V}$ by $\Sigma^{V} X$ (with $G$ acting diagonally), then we can form the infinite loop space colim ${ }_{V \subset \mathscr{U}} \Omega^{V} \Sigma^{V} X$. In general, an equivariant infinite loop space is a space $X$ such that, for every finite-dimensional $G$-invariant $V \subset \mathscr{U}$, there is a $G$-space $Y(V)$ and a $G$-homeomorphism $X \cong \Omega^{V} Y(V)$; moreover there should be compatible relations $Y(V) \cong \Omega^{W} Y(V \oplus W)$ when $V$ and $W$ are perpendicular. We call $Y(V)$ the $V$ th delooping of $X$. Such infinite loop spaces often are given in the form colim $\operatorname{li\mathscr {U}}^{V} T(V)$ for a collection of $G$-spaces $T(V)$ possessing structure maps $T(V) \rightarrow \Omega^{W} T(V \oplus W)$ for $V$ and $W$ perpendicular. (Of course, what we are really talking about here are the spectra and prespectra of [LMS].) 
In $\S \S 2-3$, we introduce $G$-operads and the associated monads that they produce. Most pertinently, we show how suitable kinds of $G$-operads give rise to monads in the category of $\mathscr{G}$-spaces. Thus a suitable $G$-operad $\mathscr{C}$ gives rise to an associated monad $C: \mathscr{G} \mathscr{T} \rightarrow \mathscr{G} \mathscr{T}$, where $\mathscr{G} \mathscr{T}$ denotes the category of (based) $\mathscr{G}$-spaces.

If $\mathscr{X}$ is a $\mathscr{G}$-space and if each constituent space $\mathscr{X}(G / K)$ is a grouplike $h$-space (in the sense that $\pi_{0}(\mathscr{X}(G / K))$ is a group), then we shall refer to $\mathscr{X}$ as grouplike. Our main result is then the following.

Theorem 1. Let $\mathscr{C}$ be a $C W E_{\infty} G$-operad.

(a) Let $X$ be a grouplike based G-space of the homotopy type of a $G-C W$ complex, and suppose that $\mathscr{C}$ acts on $X$. Then $X$ is $G$-homotopy equivalent to an equivariant infinite loop space.

(b) Let $\mathscr{X}$ be a grouplike based $\mathscr{G}$-space such that each constituent space $\mathscr{X}(G / H)$ has the homotopy type of a $C W$ complex, and suppose that $\mathscr{C}$ acts on $\mathscr{X}$. Then there exists an equivariant infinite loop space $X$ such that $\Phi X$ is homotopy equivalent to $\mathscr{X}$ as a $\mathscr{G}$-space.

Here $\Phi$ is the functor which associates to any $G$-space its system of fixedpoint spaces. Theorem 1(b), in association with suitable models for the fixed subsets of equivariant $B F$, then leads to:

Corollary 2. Equivariant $B F$ is G-homotopy equivalent to an equivariant infinite loop space.

In addition, we give the promised construction of equivariant EilenbergMac Lane spectra, thereby providing an alternative construction of the ordinary $R O(G)$-graded theory [LMM].

The paper is arranged as follows. In $\S 2$ we introduce $G$-operads and monads, and discuss the pertinent examples. $\mathscr{G}$-spaces and monads in the category of $\mathscr{G}$-spaces are discussed in $\S 3$. $\S 4$ discusses the relationship between the two monads, one in the category of $G$-spaces and the other in the category of $\mathscr{G}$ spaces, associated with a $G$-operad. We prove Theorem 1 in $\S 5$. $\S 6$ and $\S 7$ give applications of Theorem 1 ; in $\S 6$ we prove that equivariant $B F$ is an infinite loop space, and in $\S 7$ we construct the equivariant Eilenberg-Mac Lane spaces associated with a Mackey functor. Finally, $\S 8$ contains the (somewhat messy) details of some constructions discussed in $\S 4$.

The authors are indebted to J. Peter May for many discussions on the subject (and in particular for his suggestion of the approach we use in $\S 4$ ), to the referee for many useful suggestions, and to Hofstra University for providing released time.

\section{G-OPERADS}

The notion of an operad is due to May [M1]; the definition of an equivariant operad is given in [LMS], and we summarize it here. Recall that, if $X$ is a left $G \times \Pi$-space, we can think of $G$ as acting on the left, and $\Pi$ as acting on the 
right of $X$; the left and right actions of $\Pi$ are related by $x \pi=\pi^{-1} x$.

Definition 2.1. A $G$-operad is a pair $(\mathscr{C}, \gamma)$, where $\mathscr{C}$ is a sequence of $G \times \Sigma_{k}$ spaces $\mathscr{C}(k), k \geq 0$ (as above, we think of $\Sigma_{k}$ as acting on the right), and $\gamma$ is a $G$-map

$$
\gamma: \mathscr{C}(k) \times \mathscr{C}\left(i_{1}\right) \times \cdots \times \mathscr{C}\left(i_{k}\right) \rightarrow \mathscr{C}\left(i_{1}+\cdots+i_{k}\right)
$$

satisfying:

(a) $\mathscr{C}(0)$ is a single point $*$, and there is a distinguished $G$-fixed point $1 \in \mathscr{C}(1)$

(b) $\gamma$ satisfies the associativity condition

$$
\gamma\left(\gamma\left(c ; d_{1}, \ldots, d_{k}\right) ; e_{1}, \ldots, e_{i}\right)=\gamma\left(c ; f_{1}, \ldots, f_{k}\right)
$$

where $i=i_{1}+\cdots+i_{k}$ and $f_{s}=\gamma\left(d_{s} ; e_{i_{1}+\cdots+i_{s-1}+1}, \ldots, e_{i_{1}+\cdots+i_{s}}\right)$, or $*$ if $i_{s}=0$;

(c) $\gamma(1 ; d)=d$ for $d \in \mathscr{C}(k)$, and $\gamma\left(c, 1^{k}\right)=c$ for $c \in \mathscr{C}(k)$;

(d) if $c \in \mathscr{C}(k), d_{s} \in \mathscr{C}\left(i_{s}\right), \sigma \in \Sigma_{k}$, and $\tau_{s} \in \Sigma_{i_{s}}$, then

$$
\gamma\left(c \sigma ; d_{\sigma(1)}, \ldots, d_{\sigma(k)}\right)=\gamma\left(c ; d_{1}, \ldots, d_{k}\right) \sigma\left(i_{1}, \ldots, i_{k}\right)
$$

and

$$
\gamma\left(\sigma ; d_{1} \tau_{1}, \ldots, d_{k} \tau_{k}\right)=\gamma\left(c ; d_{1}, \ldots, d_{k}\right)\left(\tau_{1} \oplus \cdots \oplus \tau_{k}\right)
$$

where, if $i=i_{1}+\cdots+i_{k}$, then $\sigma\left(i_{1}, \ldots, i_{k}\right) \in \Sigma_{i}$ permutes $i$ letters in blocks as $\sigma$ permutes $k$ letters, and $\tau_{1} \oplus \cdots \oplus \tau_{k} \in \Sigma_{i}$ is the evident permutation.

We shall refer simply to a $G$-operad $\mathscr{C}$ if the maps $\gamma$ are clear from the context. The following examples are the most important for us.

Examples 2.2. (a) Let $\mathscr{U}$ be an infinite-dimensional real $G$-inner product space. Let $\mathscr{L}(k)=\mathscr{I}\left(\mathscr{U}^{k}, \mathscr{U}\right)$ be the space of (nonequivariant) linear isometries $\mathscr{U}^{k} \rightarrow \mathscr{U}$, with $\Sigma_{k}$ acting through the obvious action on $\mathscr{U}^{k}$, and $G$ acting by conjugation via the diagonal action on $\mathscr{U}^{k}$. The structure maps $\gamma$ are given by composition, and $1 \in \mathscr{L}(1)$ is the identity map.

(b) Let $V$ be a finite-dimensional real $G$-inner product space, and let $D(V)$ be the unit disc in $V$. A little disc in $V$ is a (nonequivariant) affine map $f: D(V) \rightarrow D(V)$ of the form

$$
f(v)=v_{0}+r v,
$$

where $v_{0} \in D(V)$ and $0<r \leq 1$. Let $\mathscr{C}_{V}(k)$ be the space of all $k$-tuples of nonoverlapping little discs in $V . \Sigma_{k}$ acts in the evident way on $k$-tuples, and $G$ acts by conjugation on little discs. The structure maps $\gamma$ are again given by composition, and $1 \in \mathscr{C}_{V}(1)$ is the identity map. This is an analogue of May's "little cubes" operad [M1], and a variant is used in [CW] to obtain approximations to equivariant loop spaces.

(c) If $\mathscr{U}$ is an infinite-dimensional real $G$-inner product space, we can define

$$
\mathscr{C}_{\mathscr{U}}(k)=\operatorname{colim}_{V} \mathscr{C}_{V}(k)
$$


where the colimit is taken over all finite-dimensional subspaces of $\mathscr{U}$. If $V \subset$ $W$, then $\mathscr{C}_{V}(k) \rightarrow \mathscr{C}_{W}(k)$ is defined by taking a little disc $f: D(V) \rightarrow D(V)$, given by $f(v)=v_{0}+r v$, to the disc $g: D(W) \rightarrow D(W)$ given by $g(w)=$ $v_{0}+r w$, considering $v_{0} \in V \subset W$. Again, this is analogous to the corresponding colimit of little cubes used in [M1].

There are several specials types of $G$-operads we shall want to refer to:

Definition 2.3. Let $\mathscr{C}$ be a $G$-operad.

(a) $\mathscr{C}$ is $C W$ if each $\mathscr{C}(k)$ has the $G$-homotopy type of a $G$-CW complex.

(b) $\mathscr{C}$ is $\Sigma$-free if $\mathscr{C}(k)$ is a free $\Sigma_{k}$-space for each $k$.

(c) $\mathscr{C}$ is complete if, for every subgroup $H \subset G$ and every epimorphism $\rho: H \rightarrow \Sigma_{k}, \mathscr{C}(k)^{\Lambda}$ is nonempty, where $\Lambda=\{(h, \rho(h)) \mid h \in H\}$.

(d) $\mathscr{C}$ is an $E_{\infty} G$-operad if $\mathscr{C}(k)$ is a universal principal $\left(G, \Sigma_{k}\right)$-bundle for each $k$, i.e., $\mathscr{C}(k)$ is $\Sigma_{k}$-free, and $\mathscr{C}(k)^{\Lambda}$ is contractible for every $\Lambda \subset$ $G \times \Sigma_{k}$ such that $\Lambda \cap \Sigma_{k}$ is trivial.

The $G$-operads $\mathscr{L}$ and $\mathscr{C}_{V}$ given above are $\Sigma$-free $\mathrm{CW} G$-operads (see [W1] for the CW condition). $\mathscr{L}$ and $\mathscr{C}_{\mathscr{U}}$ are both $E_{\infty} G$-operads when $\mathscr{U}$ is a complete $G$-universe, i.e., $\mathscr{U}$ contains infinitely many copies of each representation of $G . \mathscr{C}_{V}$ is complete if $V$ is large enough; precisely, $V$ must be so large that there are $G$-embeddings in $V$ of all of the orbits $G / H$.

As in [M1 and LMS], a $G$-operad $\mathscr{C}$ gives rise to a monad $C$ in the category $G \mathscr{T}$ of based $G$-spaces. If $X$ is a based $G$-space, we define

$$
C X=\coprod_{k} \mathscr{C}(k) \times_{\Sigma_{k}} X^{k} / \sim,
$$

where $\sim$ is the identification given by

$$
\left[c ; *, x_{2}, \ldots, x_{k}\right] \sim\left[\gamma(c ; *, 1, \ldots, 1) ; x_{2}, \ldots, x_{k}\right] .
$$

The term "monad" refers to the presence of natural transformations $\mu: C C X \rightarrow$ $C X$ and $\varepsilon: X \rightarrow C X$, coming from the operad structure of $\mathscr{C}$, making the following diagrams commute:

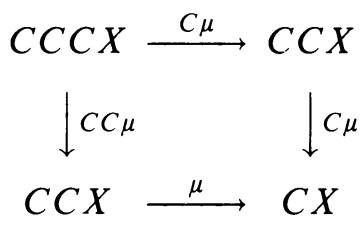

and

$$
\begin{gathered}
C X \stackrel{C_{\varepsilon}}{\longrightarrow} C C X \stackrel{\varepsilon}{\longleftarrow} C X \\
=\searrow \underset{\downarrow \mu}{\downarrow} /= \\
C X .
\end{gathered}
$$

Definition 2.4. A based $G$-space $X$ is a $C$-space if there is given a based $G$ map $\nu: C X \rightarrow X$ satisfying the associative and unital axioms obtained from 
the diagrams above by deleting one copy of $C$. We also say that the operad $\mathscr{C}$ acts on the space $X$. If $\mathscr{C}$ is an $E_{\infty}-G$-operad, we call $X$ an $E_{\infty}-G$-space.

Finally, since we are interested in fixed-point information, we consider the space $(C X)^{H}$, where $H \subset G$, in the case where $\mathscr{C}$ is $\Sigma$-free. If $k \geq 1$, let $\bar{k}=\{1, \ldots, k\}$, and let $\overline{0}=\varnothing$. Let $\Sigma_{k}$ act on $\bar{k}$ on the right in the usual way; if we wish it to act on the left we let $\sigma i=i \sigma^{-1}$. Then $X^{k}=F(\bar{k}, X)$ as a $G \times \Sigma_{k}$-space, where $F(-,-)$ denotes the space of all functions. Let

$$
\pi_{k}: \mathscr{C}(k) \times_{\Sigma_{k}} \bar{k} \rightarrow \mathscr{C}(k) / \Sigma_{k}
$$

denote the projection. Assuming that $\mathscr{C}$ is $\Sigma$-free, this is a $G$-fiber bundle with fiber $\bar{k}$. We think of the bundle $\mathscr{C}(k) \times_{\Sigma_{k}} X^{k}=\mathscr{C}(k) \times_{\Sigma_{R}} F(\bar{k}, X)$ as the space of maps from the fibers $\pi_{k}^{-1}(c)$ into $X$ (thus specifying the topology on that space of maps). If $\zeta \in\left(\mathscr{C}(k) \times_{\Sigma_{k}} X^{k}\right)^{H}$, then $\zeta$ sits over an $H$-fixed point $c \in\left(\mathscr{C}(k) / \Sigma_{k}\right)^{H}$. This gives an action of $H$ on $\pi_{k}^{-1}(c)$, and $\zeta$ gives an $H$-map from $\pi_{k}^{-1}(c)$ into $X$. Conversely, if $c \in\left(\mathscr{C}(k) / \Sigma_{k}\right)^{H}$ and if $\zeta$ is any $H$-map from $\pi_{k}^{-1}(c)$ into $X$, then $\zeta$ gives an $H$-fixed point in $\left(\mathscr{C}(k) \times_{\Sigma_{k}} F(\bar{k}, X)\right)^{H}$. Topologizing the space of all $H$-maps from fibers $\pi_{k}^{-1}(c)$ into $X, c$ ranging over the $H$-fixed points of $\mathscr{C}(k) / \Sigma_{k}$, as a subspace of $\mathscr{C}(k) \times_{\Sigma_{k}} F(\bar{k}, X)$, we record these observations in the following lemma:

Lemma 2.5. If $(\mathscr{C}, \gamma)$ is $\Sigma$-free, then $\left(\mathscr{C}(k) \times_{\Sigma_{k}} X^{k}\right)^{H}$ is homeomorphic to the space of $H$-maps from the various fibers $\pi_{k}^{-1}(c)$ to $X$, where $c$ ranges over the points of $\left(C(k) / \Sigma_{k}\right)^{H}$.

Thus we can (and often shall) think of points $f \in(C X)^{H}$ as represented by pairs $(c, \zeta)$ where $c \in\left(\mathscr{C}(k) / \Sigma_{k}\right)^{H}$, and $\zeta: \pi_{k}^{-1}(c) \rightarrow X$ is an $H$-map. These pairs are taken modulo the equivalence relation inherited from the definition of $C X$.

\section{ACTIONS OF OPERADS ON $\mathscr{G}$-SPACES}

We wish to construct certain equivariant infinite loop spaces by specifying their fixed-point data and then applying a coalescence construction. We start by recalling, from [E], Elmendorf's machinery for such a construction.

Let $\mathscr{G}$ be the category whose objects are the (left) $G$-sets $G / H$, for $H \subset G$, and whose morphisms are the $G$-maps.

Definition 3.1. A based $\mathscr{G}$-space $\mathscr{Z}$ is a contravariant functor $\mathscr{G} \rightarrow \mathscr{T}$, where $\mathscr{T}$ is the category of based spaces. A map of based $\mathscr{G}$-spaces is a natural transformation of such functors. The category of based $\mathscr{G}$-spaces will be called $\mathscr{G} \mathscr{T}$. We shall call a map $\eta$ in $\mathscr{G} \mathscr{T}$ a weak $\mathscr{G}$-equivalence if $\eta(G / H)$ is a (nonequivariant) weak equivalence for each $H \subset G$. 
If $X$ is any based $G$-space, define a $\mathscr{G}$-space $\Phi X$ by letting $\Phi X(G / H)=$ $X^{H} \cong F_{G}(G / H, X)$, where $F_{G}(-,-)$ denotes the space of $G$-maps. This clearly defines a functor $\Phi: G \mathscr{T} \rightarrow \mathscr{G} \mathscr{T}$, where $G \mathscr{T}$ is the category of based $G$-spaces and $G$-maps.

In [E], Elmendorf constructs a "coalescing" functor $E: \mathscr{G} \mathscr{T} \rightarrow G \mathscr{T}$. This functor has the property that there are natural transformations $\eta: E \Phi X \rightarrow X$ and $\varepsilon: \Phi E \mathscr{X} \rightarrow \mathscr{X}$ such that $\eta$ is a weak $G$-equivalence and $\varepsilon$ is a weak $\mathscr{G}$-equivalence. Further, on passage to the appropriate homotopy categories, $E$ becomes a right adjoint to $\Phi$.

Thus, to construct a $G$-space with specified fixed-point behavior, we can construct a $\mathscr{G}$-space and then apply $E$. Since $E$ does strange things to the geometry of the spaces involved, we would like a criterion in terms of $\mathscr{X}$, whose spaces may be simple, that will tell us that $E \mathscr{X}$ will be (equivalent to) an equivariant infinite loop space. Towards this end, we describe how a $G$ operad gives rise to a monad in the category $\mathscr{G} \mathscr{T}$. We shall only do this for $\Sigma$-free operads, but this will suffice for our purposes.

As motivation for the following construction, we want the monad $C$ in $\mathscr{G} \mathscr{T}$, associated with the $G$-operad $\mathscr{C}$, to be compatible with the monad $C$ in $G \mathscr{T}$. That is, if $X$ is a $G$-space, then we should have $C \Phi X \cong \Phi C X$; using Lemma 2.5 , we can write down what $\Phi C X$ is. This essentially forces the construction; this property of the construction will be recorded in Proposition 4.1.

Let $\mathscr{Z}$ be a based $\mathscr{G}$-space, and let $H \subset G$. Then we can consider $\bigvee_{K \subset H^{\prime}} \mathscr{X}(G / K)$ to be an $H$-space in the following way. Let $h \in H$ and $K \subset H$, and let $h^{\wedge}: G / h^{-1} K h \rightarrow G / K$ be the $G$-map given by $h^{\wedge}\left(g h^{-1} K h\right)=g h^{-1} K$. Since $\left(h_{1} h_{2}\right)^{\wedge}=\hat{h_{2}} \hat{h_{1}}$, we have $\mathscr{X}\left(\left(h_{1} h_{2}\right)^{\wedge}\right)=\mathscr{X}\left(\hat{h_{1}}\right) \mathscr{X}\left(h_{2}\right)$. Letting $h$ act on the wedge via $\mathscr{X}\left(h^{\wedge}\right)$, we then obtain the desired left $H$-action.

Now let $\mathscr{C}$ be a $\Sigma$-free $G$-operad, and let $\mathscr{Z}$ be a $\mathscr{G}$-space. We construct a $\mathscr{G}$-space $C \mathscr{Z}$. Referring to Lemma 2.5 , let

$$
C \mathscr{X}(G / H) \subset\left[C\left(\bigvee_{K \subset H} \mathscr{X}(G / K)\right)\right]^{H}
$$

be the subspace consisting of those $H$-maps $\zeta: \pi_{k}^{-1}(c) \rightarrow \bigvee_{K \subset H} \mathscr{Z}(G / K)$ such that $\zeta(y) \in \mathscr{X}\left(G / H_{y}\right)$, where $H_{y}$ is the isotropy group of $y$ under the action of $H$ on the fiber $\pi_{k}^{-1}(c)$.

Proposition 3.2. $C$ as defined above is a monad in $\mathscr{G} \mathscr{T}$.

Proof. First we must verify that $C \mathscr{X} \in \mathscr{G} \mathscr{T}$, for which we must define $C \mathscr{Z}(f)$, for $f: G / J \rightarrow G / H$. Suppose that $f(g J)=g g_{0} H$, and let $\zeta: \pi_{k}^{-1}(c) \rightarrow$ $\bigvee_{K \subset H} \mathscr{X}(G / K)$ be an $H$-map representing an element of $C \mathscr{X}(G / H)$. Then 
we let $C \mathscr{Z}(f)(\zeta)$ be the composite

$$
\begin{aligned}
\pi_{k}^{-1}\left(g_{0} c\right) \stackrel{\alpha}{\rightarrow} \pi_{k}^{-1}(c) \stackrel{\zeta^{\prime}}{\rightarrow} \bigvee_{y \in \pi_{k}^{-1}(c)} \mathscr{X}\left(G / H_{y}\right) \\
\stackrel{\beta}{\rightarrow} \bigvee_{y^{\prime} \in \pi_{k}^{-1}\left(g_{0} c\right)} \mathscr{X}\left(G / J_{y^{\prime}}\right) \stackrel{\Delta}{\rightarrow} \bigvee_{K \subset J} \mathscr{X}(G / K)
\end{aligned}
$$

where

(i) $\alpha\left(y^{\prime}\right)=g_{0}^{-1} y^{\prime}$;

(ii) $\zeta^{\prime}$ is $\zeta$ with the obvious change of range;

(iii) $\beta$ is the wedge of maps induced by the $G$-maps

$$
G / J_{y^{\prime}}=G / J_{g_{0} y} \stackrel{f^{\prime}}{\longrightarrow} G /\left(g_{0}^{-1} J g_{0}\right)_{y} \rightarrow G / H_{y},
$$

where $f^{\prime}\left(g J_{g_{0} y}\right)=g g_{0}\left(g_{0}^{-1} J g_{0}\right)_{y}$, and the last map is the projection induced by the inclusion $g_{0}^{-1} J g_{0} \subset H$;

(iv) $\Delta$ is the folding map.

$C \mathscr{X}(f)$ so defined is independent of the choice of $g_{0}$, and functorial in $f$, making $C \mathscr{X}$ a $\mathscr{G}$-space.

As for the monad structure, we need natural transformations $\mu: C C \mathscr{Z} \rightarrow$ $C \mathscr{X}$ and $\varepsilon: \mathscr{X} \rightarrow C \mathscr{X} . \mu$ is constructed as follows. Suppose that

$$
\zeta: \pi_{k}^{-1}(c) \rightarrow \bigvee_{K \subset H} C \mathscr{X}(G / K)
$$

is an $H$-map representing a point of $C C \mathscr{X}(G / H)$. Then, for each $y \in \pi_{k}^{-1}(c)$,

$$
\zeta(y): \pi_{i_{y}}^{-1}\left(c_{y}\right) \rightarrow \bigvee_{K \subset H_{y}} \mathscr{X}(G / K)
$$

is an $H_{y}$-map. Let $i=\Sigma_{y \in \pi_{k}^{-1}(c)} i_{y}$; the structure map of the operad gives us a point $d=\gamma\left(\tilde{c} ;\left(\tilde{c}_{y}\right)\right) \Sigma_{i} \in \mathscr{C}(i) / \Sigma_{i}$, where $\tilde{c} \in \mathscr{C}(k)$ is any point over $c$, and so forth. Moreover, the maps $\zeta(y)$ assemble together to give an $H$-map

$$
\mu(\zeta): \pi_{i}^{-1}(d) \rightarrow \bigvee_{K \subset H} \mathscr{X}(G / H)
$$

representing an element of $C \mathscr{X}(G / H)$.

$\varepsilon$ is constructed by sending $x \in \mathscr{X}(G / H)$ to the function $\zeta: \pi_{1}^{-1}(1) \rightarrow$ $C \mathscr{X}(G / H)$ that takes the single point in the domain to $x$. That $\mu$ and $\varepsilon$ satisfy the axioms for a monad can now be checked, using the properties of the $G$-operad $\mathscr{C}$.

Having the monad $C$, we can define a $C$-object in $\mathscr{G} \mathscr{T}$, or a $C$ - $\mathscr{G}$-space, to be a $\mathscr{G}$-space $\mathscr{X}$ with a $\mathscr{G}$-map $\nu: C \mathscr{X} \rightarrow \mathscr{X}$ satisfying the usual axioms. If $\mathscr{X}$ is a $C-\mathscr{G}$-space, then it follows from the definition that each space $\mathscr{X}(G / H)$ is an $h$-space, and that the structure maps $\mathscr{X}(G / H) \rightarrow \mathscr{X}(G / K)$ are $h$-maps. 
We should also comment that it can be shown, as in [M1], that the monads $C$ that we have constructed in $G \mathscr{T}$ and $\mathscr{G} \mathscr{T}$ both preserve weak equivalences.

\section{CoAlesCence AND MONADS}

Given a $\Sigma$-free $G$-operad $\mathscr{C}$, we have thus far defined two monads, $C: G \mathscr{T}$ $\rightarrow G \mathscr{T}$ and $C: \mathscr{G} \mathscr{T} \rightarrow \mathscr{G} \mathscr{T}$. We also have the functor $\Phi: G \mathscr{T} \rightarrow \mathscr{G} \mathscr{T}$ and we now comment that the two monads correspond via this functor.

Proposition 4.1. There is a natural homeomorphism $\Phi C \cong C \Phi$. Moreover, the following diagrams commute:

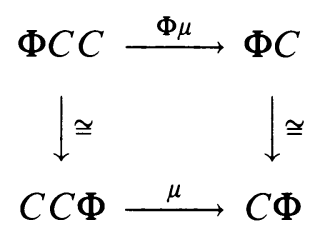

and

$$
\begin{gathered}
\Phi \stackrel{\Phi \varepsilon}{\longrightarrow} \Phi C \\
\varepsilon \searrow \quad \swarrow \cong \\
C \Phi
\end{gathered}
$$

Proof. With a bit of work, using Lemma 2.5, this is straightforward from the constructions.

We would like a similar relationship involving a coalescence functor. The details of this are a bit involved, and so are left to the last section, $\S 8$, but we state the results here. For each $G$-operad $\mathscr{C}$, we shall, using a variant of the technique in [E], construct a functor $E_{\mathscr{C}}: \mathscr{G} \mathscr{T} \rightarrow G \mathscr{T}$ and a natural map $\eta: \Phi E_{\mathscr{C}} \mathscr{X} \rightarrow \mathscr{X}$, which will be a $\mathscr{G}$-equivalence if $\mathscr{C}$ is complete. This construction will be covariant in $\mathscr{C}$. Moreover, we shall have

Proposition 4.2. There is a natural G-map $\lambda_{\mathscr{C}}: E_{\mathscr{C}} C \rightarrow C E_{\mathscr{C}}$ which is a $G$ equivalence if $\mathscr{C}$ is complete. Moreover, the following diagrams commute:

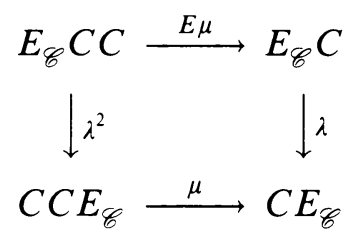

and

$$
\begin{gathered}
E_{\mathscr{C}} \stackrel{E \varepsilon}{\longrightarrow} E_{\mathscr{C}} C \\
\varepsilon \searrow \swarrow \lambda \\
C E_{\mathscr{C}} .
\end{gathered}
$$




\section{5. $E_{\infty}$-SPACES ARE EQUIVARIANT INFINITE LOOP SPACES}

We first recall the geometric bar construction, as given in [M1]. Let $C$ and $D$ be monads in $G \mathscr{T}$, suppose that there is a map of monads $D \rightarrow C$, and suppose that $X$ is a $D$-G-space. Then we can form a simplicial $G$-space $B_{*}(C, D, X)$ by letting $B_{n}(C, D, X)=C D^{n} X$, with face and degeneracy maps given by the monad structures (for details, see [M1]). We call the geometric realization of this $B(C, D, X)$.

Similarly, if $C$ and $D$ are monads in $\mathscr{G} \mathscr{T}, C$ coming from a $G$-operad $\mathscr{C}$, and if $\mathscr{X}$ is a $D-\mathscr{G}$-space, we can define simplicial $G$-spaces $B_{*}\left(E_{\mathscr{C}} C, D, \mathscr{Z}\right)$ and $B_{*}\left(C E_{\mathscr{C}}, D, \mathscr{X}\right)$, with

$$
B_{n}\left(E_{\mathscr{C}} C, D, \mathscr{X}\right)=E_{\mathscr{C}} C D^{n} \mathscr{X} \text { and } B_{n}\left(C E_{\mathscr{C}}, D, \mathscr{X}\right)=C E_{\mathscr{C}} D^{n} \mathscr{X} \text {. }
$$

The simplicial structure on $B_{*}\left(E_{\mathscr{C}} C, D, \mathscr{Z}\right)$ is the evident one, and the structure on $B_{*}\left(C E_{\mathscr{C}}, D, \mathscr{X}\right)$ uses the map $C E_{\mathscr{C}} D \rightarrow C E_{\mathscr{C}} C \rightarrow C C E_{\mathscr{C}} \rightarrow C E_{\mathscr{C}}$ coming from Proposition 4.2. If $\mathscr{C}$ is complete, $\lambda_{\mathscr{C}}$ induces a $G$-equivalence

$$
B(\lambda, 1,1): B\left(E_{\mathscr{C}} C, D, \mathscr{X}\right) \rightarrow B\left(C E_{\mathscr{C}}, D, \mathscr{X}\right) \text {. }
$$

We may also form bar constructions in the category $\mathscr{G} \mathscr{T}$. For example, we can form the $\mathscr{G}$-space $B(C, D, \mathscr{Z})$, constructed from the simplicial $\mathscr{G}$-space $B_{n}(C, D, \mathscr{Z})=C D^{n} \mathscr{X}$.

We now prove Theorem 1 by a sequence of lemmas.

Lemma 5.1. If $\mathscr{C}$ is complete and $D \rightarrow C$ is an equivalence of monads, then there is an equivalence $\Phi B\left(E_{\mathscr{C}} C, D, \mathscr{X}\right) \simeq \mathscr{X}$.

Proof. Consider the maps

$$
\begin{aligned}
\Phi B & \left(E_{\mathscr{C}} C, D, \mathscr{X}\right) \\
& =B\left(\Phi E_{\mathscr{C}} C, D, \mathscr{X}\right) \stackrel{\alpha}{\rightarrow} B(C, D, \mathscr{X}) \stackrel{\beta}{\leftarrow} B(D, D, \mathscr{X}) \stackrel{\varepsilon}{\rightarrow} \mathscr{X} .
\end{aligned}
$$

$\alpha$ is the equivalence induced by the natural equivalence $\Phi E_{\mathscr{C}} \mathscr{Y} \rightarrow \mathscr{Y}$ given in Proposition $8.1, \beta$ is induced by the equivalence $D \rightarrow C$, and $\varepsilon$ is induced by the action map $\nu^{n+1}: D^{n+1} \mathscr{X} \rightarrow \mathscr{X} . \varepsilon(G / H): B(D, D, \mathscr{X})(G / H) \rightarrow \mathscr{X}(G / H)$ is a deformation retraction for each $H$, by the usual simplicial argument [M1, $\S 9$ and $\S 11]$. Since these three maps are all equivalences, the result follows.

We say that a $G$-map is a group completion if it is a nonequivariant group completion on all fixed-sets. See [S or M3] for discussions of group completion. Let $Q_{\infty}$ be the monad in $G \mathscr{T}$ defined by $Q_{\infty} X=\operatorname{colim}_{V} \Omega^{V} \Sigma^{V} X$. Let $C_{V}$ and $C_{\mathscr{U}}$ be the monads associated with the $G$-operads $\mathscr{C}_{V}$ and $\mathscr{C}_{\mathscr{U}}$ of Examples 2.2 (b) and (c).

Lemma 5.2. There are compatible maps of monads $\kappa: C_{V} \rightarrow \Omega^{V} \Sigma^{V}$ such that the colimit $\kappa: C_{\mathscr{U}} \rightarrow Q_{\infty}$ is a group completion.

The construction of the maps is simple; for the proof of the group completion result see $[\mathrm{CW}]$. For the next result, write $E_{\mathscr{U}}$ for $E_{\mathscr{C}_{\mathscr{L}}}$. 
Lemma 5.3. Let $D \rightarrow C_{\mathscr{U}}$ be a map of monads, and let $X$ be a $D$-G-space. Then $B\left(C_{\mathscr{U}}, D, X\right) \rightarrow B\left(Q_{\infty}, D, X\right)$ is a group completion. Similarly, if $\mathscr{X}$ is a $D$ $\mathscr{G}$-space, then $B\left(C_{\mathscr{U}} E_{\mathscr{U}}, D, \mathscr{X}\right) \rightarrow B\left(Q_{\infty} E_{\mathscr{U}}, D, \mathscr{X}\right)$ is a group completion.

Proof. Since taking fixed-sets commutes with geometric realization, we can reduce to the nonequivariant case, where we appeal to [M3, Theorem 3.1], which shows that the geometric realization of a group completion is a group completion.

The following step was first shown by Hauschild, but has not yet appeared in print. We first need a lemma about equivariant geometric realization.

Lemma 5.4. If $K_{*}$ is a simplicial G-space, and if $X$ is an unbased G-CW complex with $\operatorname{dim} X^{J} \leq \operatorname{conn} K_{n}^{J}$ for all $n$ and $J$, then the natural map

$$
\left|F\left(X, K_{*}\right)\right| \rightarrow F\left(X,\left|K_{*}\right|\right)
$$

is a weak G-equivalence, where $|-|$ denotes geometric realization.

Proof. Let $H \subset G$; we show that $\left|F\left(X, K_{*}\right)\right|^{H} \rightarrow F\left(X,\left|K_{*}\right|\right)^{H}$ is a weak equivalence, by induction on the skeleta of $X$. At the beginning of the induction, $\left|F_{H}\left(\left(X^{0}\right)^{+}, K_{*}\right)\right| \rightarrow F_{H}\left(\left(X^{0}\right)^{+}, \mid K_{*}\right)$ (where "+ " denotes the addition of a disjoint basepoint, and $F_{H}(-,-)$ is the space of $H$-equivariant based maps) is an equivalence since geometric realization commutes with products. Now consider the fibration sequence

$$
F_{H}\left(X^{r+1} / X^{r}, K_{n}\right) \rightarrow F_{H}\left(\left(X^{r+1}\right)^{+}, K_{n}\right) \rightarrow F_{H}\left(\left(X^{r}\right)^{+}, K_{n}\right) .
$$

The base space of this fibration is connected, because, by assumption, $X^{r}$ has only cells of dimension smaller than the connectivity of $K_{n}$. By [M1, 12.7], it follows that

$$
\left|F_{H}\left(X^{r+1} / X^{r}, X_{*}\right)\right| \rightarrow\left|F_{H}\left(\left(X^{r+1}\right)^{+}, K_{*}\right)\right| \rightarrow\left|F_{H}\left(\left(X^{r}\right)^{+}, K_{*}\right)\right|
$$

is a quasifibration. We now have a commutative diagram

$$
\begin{gathered}
\left|F_{H}\left(X^{r+1} / X^{r}, K_{*}\right)\right| \longrightarrow\left|F_{H}\left(\left(X^{r+1}\right)^{+}, K_{*}\right)\right| \longrightarrow\left|F_{H}\left(\left(X^{r}\right)^{+}, K_{*}\right)\right| \\
\downarrow \\
F_{H}\left(X^{r+1} / X^{r},\left|K_{*}\right|\right) \longrightarrow F_{H}\left(\left(X^{r+1}\right)^{+},\left|K_{*}\right|\right) \longrightarrow F_{H}\left(\left(X^{r}\right)^{+},\left|K_{*}\right|\right)
\end{gathered}
$$

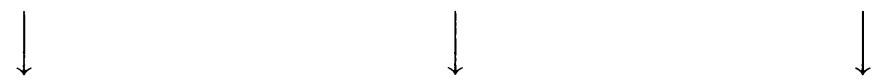

of quasifibrations, where the vertical arrows are inclusions. The rightmost arrow is a weak equivalence by induction. As for the leftmost arrow, $X^{r+1} / X^{r}$ is a wedge of spheres $G / J^{+} \wedge S^{r+1}$, so we can reduce to the nonequivariant case, and appeal to [M1, 12.3]. Thus the middle arrow is an equivalence, giving us the inductive step; since $X$ is, by assumption, finite dimensional, this completes the argument.

Suppose now that $D$ is a monad with a map of monads $D \rightarrow \Omega^{V} \Sigma^{V}$. Then we have a natural map $\Sigma^{V} D \rightarrow \Sigma^{V} \Omega^{V} \Sigma^{V} \rightarrow \Sigma^{V}$, allowing us to form the space 
$B\left(\Sigma^{V}, D, X\right)$ if $X$ is a $D$-space. Similarly, letting $E_{V}=E_{\mathscr{C}_{V}}$, if we have a natural map $E_{V} D \rightarrow \Omega^{V} \Sigma^{V} E_{V}$, we can form the space $B\left(\Sigma^{V} E_{V}, D, \mathscr{X}\right)$ when $\mathscr{Z}$ is a $D-\mathscr{G}$-space.

Lemma 5.5. The inclusions

$$
B\left(\Omega^{V} \Sigma^{V}, D, X\right) \rightarrow \Omega^{V} B\left(\Sigma^{V}, D, X\right)
$$

and

$$
B\left(\Omega^{V} \Sigma^{V} E_{V}, D, \mathscr{X}\right) \rightarrow \Omega^{V} B\left(\Sigma^{V} E_{V}, D, \mathscr{X}\right)
$$

are weak G-equivalences.

Proof. Let $K_{n}$ be either $B_{n}\left(\Sigma^{V}, D, X\right)=\Sigma^{V} D^{n} X$ or $B_{n}\left(\Sigma^{V} E_{V}, D, \mathscr{Z}\right)=$ $\Sigma^{V} E_{V} D^{n} \mathscr{X}$. Then $K_{n}^{J}$ is $\left(\left|V^{J}\right|-1\right)$-connected for each $n$ and each $J \subset G$. Let $H \subset G$ and consider the fibration sequence

$$
F_{H}\left(S^{V}, K_{n}\right) \rightarrow F_{H}\left(D(V)^{+}, K_{n}\right) \rightarrow F_{H}\left(S(V)^{+}, K_{n}\right) .
$$

The base space of this fibration is connected, because $S(V)$, considered as an $H$-CW complex, has only cells of dimension smaller than the connectivity of $K_{n}$. By [M1, 12.7], it again follows that

$$
\left|F_{H}\left(S^{V}, K_{*}\right)\right| \rightarrow\left|F_{H}\left(D(V)^{+}, K_{*}\right)\right| \rightarrow\left|F_{H}\left(S(V)^{+}, K_{*}\right)\right|
$$

is a quasifibration. We now have a commutative diagram

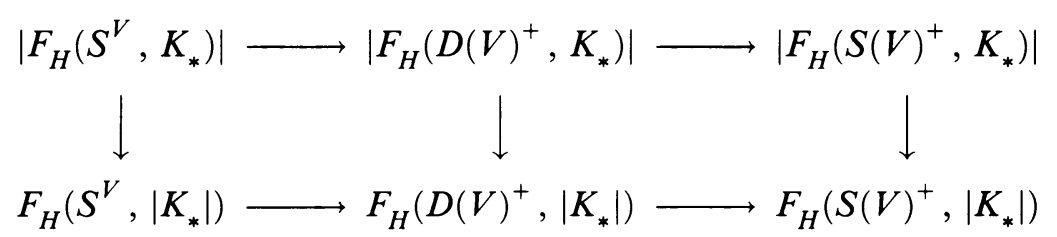

of quasifibrations. The rightmost arrow is a weak equivalence by the previous lemma. Since $D(V)$ is equivariantly contractible, the middle vertical arrow above is also an equivalence, thus the leftmost vertical arrow is an equivalence, as desired.

Proof of Theorem 1. The following arguments mimic [M3, §VII.3]. Let $\mathscr{C}$ be an $E_{\infty}-G$-operad, let $X$ be a $C$-G-space, and let $\mathscr{X}$ be a $C$ - $\mathscr{G}$-space. Let $\mathscr{D}_{V}=\mathscr{C}_{V} \times \mathscr{C}$, and let $\mathscr{D}_{V}$ act on $X$ and $\mathscr{X}$ via projection to $\mathscr{C}$. Write $D_{V}$ for the monad (in either $G \mathscr{T}$ or $\mathscr{G} \mathscr{T}$ ) associated with $\mathscr{D}_{V}$.

We first prove part (a) of the theorem. Start by noticing that there is an inclusion $X \rightarrow B\left(D_{V}, D_{V}, X\right)$, and a deformation retraction $B\left(D_{V}, D_{V}, X\right) \rightarrow X$ [M1].

Since $\mathscr{C}$ is an $E_{\infty}$-operad, the projection $\mathscr{D}_{V} \rightarrow \mathscr{C}_{V}$ is an equivalence of operads, and so $D_{V} \rightarrow C_{V}$ is a weak equivalence of monads. Therefore, $B\left(D_{V}, D_{V}, X\right) \rightarrow B\left(C_{V}, D_{V}, X\right)$ is a weak equivalence.

Since $\operatorname{colim}_{V} B\left(C_{V}, D_{V}, X\right) \cong B\left(C_{\mathscr{U}}, D_{\mathscr{U}}, X\right)$ and

$$
\operatorname{colim}_{V} B\left(\Omega^{V} \Sigma^{V}, D_{V}, X\right) \cong B\left(Q_{\infty}, D_{\mathscr{U}}, X\right),
$$


Lemma 5.3 implies that the map

$$
\operatorname{colim}_{V} B\left(C_{V}, D_{V}, X\right) \rightarrow \operatorname{colim}_{V} B\left(\Omega^{V} \Sigma^{V}, D_{V}, X\right)
$$

is an equivalence.

Putting these facts together with Lemma 5.5, we see that the $G$-map

$$
\begin{aligned}
X & \rightarrow \underset{V}{\operatorname{colim}} B\left(D_{V}, D_{V}, X\right) \rightarrow \underset{V}{\operatorname{colim}} B\left(C_{V}, D_{V}, X\right) \\
& \rightarrow \operatorname{colim} B\left(\Omega^{V} \Sigma^{V}, D_{V}, X\right) \rightarrow \underset{V}{\operatorname{colim}} \Omega^{V} B\left(\Sigma^{V}, D_{V}, X\right)
\end{aligned}
$$

is a group completion. If $X$ and $\mathscr{C}$ have the homotopy types of $G-\mathrm{CW}$ complexes, and if $X$ is grouplike, then we conclude that $X$ is $G$-homotopy equivalent to $\operatorname{colim}_{V} \Omega^{V} B\left(\Sigma^{V}, D_{V}, X\right)$, and so is an equivariant infinite loop space.

Part (b) is similar: Consider the composite

$$
\begin{aligned}
& \underset{V}{\operatorname{colim}} B\left(E_{V} C_{V}, D_{V}, \mathscr{X}\right) \rightarrow \underset{V}{\operatorname{colim}} B\left(C_{V} E_{V}, D_{V}, \mathscr{X}\right) \\
& \rightarrow \underset{V}{\operatorname{colim}} B\left(\Omega^{V} \Sigma^{V} E_{V}, D_{V}, \mathscr{X}\right) \\
& \rightarrow \underset{V}{\operatorname{colim}} \Omega^{V} B\left(\Sigma^{V} E_{V}, D_{V}, \mathscr{X}\right) \text {. }
\end{aligned}
$$

Again, this is a group completion by Proposition 4.2 and Lemmas 5.3 and 5.5. On the other hand, Lemma 5.1 shows that $\Phi \operatorname{colim}_{V} B\left(E_{V} C_{V}, D_{V}, \mathscr{X}\right) \simeq \mathscr{X}$, so if $\mathscr{X}$ and $\mathscr{C}$ are $\mathrm{CW}$, and $\mathscr{X}$ is grouplike, colim $\Omega_{V}^{V} B\left(\Sigma^{V} E_{V}, D_{V}, \mathscr{X}\right)$ is an equivariant infinite loop space with fixed-point system homotopy equivalent to $\mathscr{X}$.

\section{EQuivariant $B F$}

We show that $B_{G} F$, the $G$-space classifying equivariant stable spherical fibrations (see [W2, 3.3]), is an equivariant infinite loop space. We do this by constructing a model $\mathscr{B}_{G} \mathscr{F} \in \mathscr{G} \mathscr{T}$ of its fixed-point system, and giving $\mathscr{B}_{G} \mathscr{F}$ the structure of an $E_{\infty}-\mathscr{G}$-space.

Fix a complete $G$-universe $\mathscr{U}$, and let $V \subset \mathscr{U}$ be a finite-dimensional $G$ invariant subspace. If $H \subset G$ is a subgroup, let $F_{H}(V)$ be the topological category whose object space ob $F_{H}(V)$ is the Grassmannian of $H$-invariant $|V|$-dimensional subspaces of $\mathscr{U} \oplus V$. The morphisms, from an object $Y$ to an object $Z$, are the $H$-homotopy equivalences $S^{Y} \rightarrow S^{Z}$ of the one-point compactifications; we topologize the morphism space $\operatorname{map} F_{H}(V)$ as a bundle over ob $F_{H}(V) \times$ ob $F_{H}(V)$ via the source and target maps. As in [M2], we can form the classifying space $B F_{H}(V)=B\left(*, F_{H}(V), *\right)$ of the category using a bar construction. It has as basepoint the subspace $0 \oplus V \subset \mathscr{U} \oplus V$.

If $f: G / K \rightarrow G / H$ is given by $f(g K)=g g_{0} H$, define $f^{*}: F_{H}(V) \rightarrow F_{K}(V)$ by sending an $H$-invariant subspace $Y$ to the subspace $g_{0} Y$, and sending a morphism $\alpha: S^{Y} \rightarrow S^{Z}$ to $g_{0} \alpha g_{0}^{-1}$. Since the bar construction is functorial, this gives a map $B\left(f^{*}\right): B F_{H}(V) \rightarrow B F_{K}(V)$. Using these maps we define a $\mathscr{G}$-space $\mathscr{B}_{G} \mathscr{F}(V)$ by $\mathscr{B}_{G} \mathscr{F}(V)(G / H)=B F_{H}(V)$. 
Lemma 6.1. The $G$-space $E \mathscr{B}_{G} \mathscr{F}(V)$ classifies $|V|$-dimensional equivariant spherical fibrations.

Proof. We prove the lemma by comparing $E \mathscr{B}_{G} \mathscr{F}(V)$ to a model for $B_{G} F$ constructed along the lines of [W2]. Referring to [W2, §2], consider the topological category $\mathscr{A}$ whose object space is the space of projections $p(H, W): G \times{ }_{H}$ $S^{W} \rightarrow G / H$, topologized as the disjoint union over the subgroups $H$ of $G$ of the Grassmannians of $H$-invariant $|V|$-dimensional planes $W$ in $\mathscr{U} \oplus V$; the morphisms from $p(H, W)$ to $p(K, Z)$ are the equivariant fiberwise basepointpreserving homotopy equivalences. There is a continuous functor $\varphi$ from $\mathscr{A}$ to the category of $G$-spaces, given by taking basespaces. The $G$-space $B(*, \mathscr{A}, \varphi)$ classifies $|V|$-dimensional spherical fibrations, by the theory of [W2].

There is a natural equivalence $\varepsilon: B(*, \mathscr{A}, \varphi)^{H} \rightarrow B F_{H}(V)$, given by the following construction on the simplicial level. An element of $B(*, \mathscr{A}, \varphi)^{H}$ can be represented by a tuple $\left(*\left[f_{n}, \cdots, f_{1}\right] \alpha\right)$ where $f_{i}: p\left(H_{i-1}, W_{i-1}\right) \rightarrow p\left(H_{i}, W_{i}\right)$ and $\alpha: G / H \rightarrow G / H_{0}$. We let $\varepsilon\left(*\left[f_{n}, \ldots, f_{1}\right] \alpha\right)=\left(*\left[\psi_{n}, \ldots, \psi_{1}\right] *\right)$; here $\psi_{i}: Z_{i-1} \rightarrow Z_{i}$, where, if the composite $\varphi\left(f_{i}\right) \circ \cdots \circ \varphi\left(f_{1}\right) \circ \alpha(e H)=g_{i} H_{i}$, then $Z_{i}=g_{i} W_{i}$, the translate of the plane $W_{i} \subset \mathscr{U} \oplus V$, and $\psi_{i}\left(g_{i} w\right)=g_{i+1} w^{\prime}$ if $f_{i}\left[g_{i}, w\right]=\left[g_{i+1}, w^{\prime}\right]$. To see that $\varepsilon$ is an equivalence for each $H$, use the techniques in [W2, 2.3.2]; show that each component of both $B(*, \mathscr{A}, \varphi)^{H}$ and $B F_{H}(V)$ is the basespace of a principal fibration with contractible total space, with $\varepsilon$ lifting to a fiberwise equivalence, and show that $\varepsilon$ gives a one-to-one correspondence of components.

Now, since $E$ is left adjoint to $\Phi[\mathrm{E}]$, we get a $G$-map $E \varepsilon: B(*, \mathscr{A}, \varphi) \rightarrow$ $E \mathscr{B}_{G} \mathscr{F}(V)$ that is an equivalence on all fixed-sets, and hence a $G$-equivalence.

If $V \subset W$, addition of $W-V$ (the orthogonal complement of $V$ in $W$ ) defines a functor $F_{H}(V) \rightarrow F_{H}(W)$ for all $H$, and so a $\mathscr{G}$-map $\mathscr{B}_{G} \mathscr{F}(V) \rightarrow$ $\mathscr{B}_{G} \mathscr{F}(W)$. Letting

$$
\mathscr{B}_{G} \mathscr{F}=\operatorname{colim}_{V} \mathscr{B}_{G} \mathscr{F}(V),
$$

we obtain a $\mathscr{G}$-space such that $B_{G} F=E \mathscr{B}_{G} \mathscr{F}$ classifies equivariant stable spherical fibrations.

Now let $\mathscr{L}$ be the linear isometries operad of Example 2.2(a), and let $L$ be the associated monad in $\mathscr{G} \mathscr{T}$. We give $\mathscr{B}_{G} \mathscr{F}$ the structure of an $\mathscr{L}-\mathscr{G}$-space. Thus we need to construct $\nu: L \mathscr{B}_{G} \mathscr{F} \rightarrow \mathscr{B}_{G} \mathscr{F}$.

An element $z \in L \mathscr{B}_{G} \mathscr{F}(G / H)$ is represented by an $H$-map

$$
\zeta: \pi_{k}^{-1}(c) \rightarrow \bigvee_{K \subset H} B F_{K}(V)
$$

for some $V$, where $\pi_{k}: \mathscr{L}(k) \times_{\Sigma_{k}} \bar{k} \rightarrow \mathscr{L}(k) / \Sigma_{k}$. Now, there is an inclusion $\mathscr{L}(k)=\mathscr{I}\left(\mathscr{U}^{k}, \mathscr{U}\right) \rightarrow \mathscr{I}(\mathscr{U}, \mathscr{U})^{k}$ which takes an isometry $\theta: \mathscr{U}^{k} \rightarrow \mathscr{U}$ to its restrictions $\left(\theta_{1}, \ldots, \theta_{k}\right)$ to the $k$ summands. This, in fact, shows $\mathscr{L}(k)$ as the subspace of $k$-tuples of mutually orthogonal isometries $\mathscr{U} \rightarrow \mathscr{U}$. The adjoint 
of this inclusion is a map $\mathscr{L}(k) \times \bar{k} \rightarrow \mathscr{I}(\mathscr{U}, \mathscr{U})$ taking $(\theta, i)$ to $\theta_{i}$. Further, this map is $\Sigma_{k}$-invariant, giving a map $\mathscr{L}(k) \times_{\Sigma_{k}} \bar{k} \rightarrow \mathscr{I}(\mathscr{U}, \mathscr{U})$. In this way, we think of the points of $\pi_{k}^{-1}(c)$ as giving $k$ mutually orthogonal isometries of $\mathscr{U}$ to itself. If $c \in\left(\mathscr{L}(k) / \Sigma_{k}\right)^{H}$, then the $H$-action by conjugation on the isometries in $\pi_{k}^{-1}(c)$ is the same as a permutation action given by a certain homomorphism $H \rightarrow \Sigma_{k}$.

With $\zeta$ representing $z$ as above, we have now to define $\mu(z) \in \mathscr{B}_{G} \mathscr{F}(G / H)$. Let $\left\{\theta_{y}\right\}_{y \in \pi_{k}^{-1}(c)}$ be the collection of isometries associated with the fiber $\pi_{k}^{-1}(c)$. Then we can define a functor

$$
\theta_{*}: \prod_{y \in \pi_{k}^{-1}(c)} F_{H_{y}}(V) \rightarrow F_{e}(W)
$$

where, because $c$ is $H$-fixed, $W=\bigoplus_{y} \theta_{y}(V)$ is an $H$-invariant subspace of $\mathscr{U}$. $\theta_{*}$ is defined by taking a collection $\left(Z_{y}\right)$ of subspaces in $\mathscr{U} \oplus V$ to the subspace $\oplus \theta_{y}\left(Z_{y}\right)$ of $\mathscr{U} \oplus W$, and it assembles morphisms similarly. Let $H$ act on the category on the left by $\left[h\left(Z_{y}\right)\right]_{y^{\prime}}=h Z_{h^{-1} y^{\prime}}$, and similarly on morphisms. Then it is not hard to see that $\theta_{*}$ restricts to a functor

$$
\theta_{*}:\left(\prod_{y} F_{H_{y}}(V)\right)^{H} \rightarrow F_{H}(W),
$$

the point being that $H$-invariant collections of subspaces assemble into $H$ invariant subspaces, and $H$-invariant collections of homotopy equivalences assemble into $H$-homotopy equivalences. Applying the functor $B$, which commutes with fixed-points and products, gives

$$
B \theta_{*}:\left(\prod_{y} B F_{H_{y}}(V)\right)^{H} \rightarrow B F_{H}(W) .
$$

Since $\zeta$ specifies a point of the space on the left, by applying $B \theta_{*}$ we obtain a point $\nu(\zeta) \in B F_{H}(W)$. Including $W$ into a $G$-invariant subspace, and passing to the colimit, we obtain $\nu(z) \in \mathscr{B}_{G} \mathscr{F}(G / H)$.

Now it is not difficult to check that $\nu$ does define a map of $\mathscr{G}$-spaces $L \mathscr{B}_{G} \mathscr{F} \rightarrow \mathscr{B}_{G} \mathscr{F}$, and that this makes $\mathscr{B}_{G} \mathscr{F}$ into an $\mathscr{L}-\mathscr{G}$-space. Since $\mathscr{L}$ is an $E_{\infty}-G$-operad and each $\mathscr{B}_{G} \mathscr{F}(G / H)$ is clearly group-like, Theorem 1 now allows us to conclude

Corollary 2. $B_{G} F$ is an equivariant infinite loop space.

This completes the argument in [HW, $\S 4]$, where it is assumed that $\mathrm{Sph}_{G}$, the spherical fibration analogue of $K$-theory, is a cohomology theory, and hence admits an equivariant transfer. This was used to prove an equivariant version of the Adams conjecture. One should be able to use $\mathrm{Sph}_{G}$ and its transfer to further study homotopy equivalence of representations; the Becker-Gottlieb 
techniques used in [HW] convert questions about such equivalences into questions about equivalences of spherical fibrations with simpler group actions.

\section{Equivariant Eilenberg-Mac Lane SPaces}

Let $T$ be a Mackey functor in the sense of Dress [D1, D2]. Then we can think of $T$ as a discrete based $\mathscr{G}$-space, with 0 as the basepoint in each $T(G / H)$. We show here that there are spaces $K(T, V)$ such that $\Phi \Omega^{V} K(T, V) \simeq T$. We do this by defining an action of the little discs operad on $T$.

Let $\mathscr{C}=\mathscr{C}_{\mathscr{Q}}$ be the little discs operad of Example 2.2(c), defined using a complete $G$-universe $\mathscr{U}$, and let $C$ be the corresponding monad in $\mathscr{G} \mathscr{T}$. Define $\nu: C T(G / H) \rightarrow T(G / H)$ as follows. A typical element of $C T(G / H)$ may be represented by an $H$-equivariant map $\zeta: S \rightarrow \bigvee_{K \subset H} T(G / K)$ where $S$ is a finite $H$-set, and $\zeta(s) \in T\left(G / H_{s}\right)$ for each $s$. Represent $S$ as a disjoint union of $H$-orbits

$$
S=H / K_{1} \amalg \cdots \amalg H / K_{r},
$$

with each $K_{i} \subset H$. Let $\nu(\zeta)=x_{1}+\cdots+x_{r}$, where $x_{i}=l_{K_{i}}^{H}\left(\zeta\left(e K_{i}\right)\right)$, where

$$
l_{K_{i}}^{H}: T\left(G / K_{i}\right) \rightarrow T(G / H)
$$

is the induction map of the Mackey functor $T$ induced by the inclusion $K_{i} \subset$ $H$. Now $\nu(\zeta)$ is independent of the choice of decomposition of $S$, by the $H$-equivariance of $\zeta$ and the properties of the induction.

Alternatively, we may think of $T$ as being a contravariant functor on the category of $G$-sets and stable $G$-maps between them [LMM]. Then $S=\pi_{k}^{-1}(c)$ can be thought of (as in $\S 6$ ) as an $H$-invariant collection of little discs, which gives us a stable $H$-map $H / H \rightarrow S$. We extend this to a stable $G$-map $G / H \rightarrow$ $G \times{ }_{H} S$ and apply the functor $T$ to get the desired induction map. That $\nu$ defines an action of $\mathscr{C}$ now follows from the functoriality of $T$.

The required spaces $K(T, V)$ are then

$$
K(T, V)=\operatorname{colim}_{W} \Omega^{W} B\left(\Sigma^{W+V} E_{W+V}, D_{W+V}, T\right),
$$

where, as before, $D_{W+V}=C_{W+V} \times C$.

\section{CoAlescence Revisited}

In this section we give the details of the construction of the coalescence functor $E_{\mathscr{C}}: \mathscr{G} \mathscr{T} \rightarrow G \mathscr{T}$, and the natural equivalence $\lambda_{\mathscr{E}}: E_{\mathscr{C}} C \rightarrow C E_{\mathscr{C}}$. The construction of $E_{\mathscr{C}}$ is similar to the construction of Elmendorf's coalescence functor [E], but modifications are necessary in order to allow the definition of $\lambda_{\mathscr{C}}$.

Suppose given a $\mathscr{G}$-space $\mathscr{Z}$ and a $\Sigma$-free $G$-operad $\mathscr{C}$. We shall construct $E_{\mathscr{C}} \mathscr{Z}$ using the categorical bar construction of [M2]. For this purpose, we construct a topological category $C^{G} \mathscr{X}$ as follows. The space of objects is the 
space $C \mathscr{Z}(G / G)$. Recall that this space is constructed by first forming the $G$-space

$$
W \mathscr{X}=\bigvee_{H \subset G} \mathscr{X}(G / H)
$$

(the action of $G$ being described in $\S 3$ ), then letting $C \mathscr{X}(G / G) \subset(C W \mathscr{X})^{G}$ be the subspace consisting of $G$-maps $\zeta: \pi_{k}^{-1}(c) \rightarrow W \mathscr{X}$ such that $\zeta(s) \in$ $\mathscr{X}\left(G / G_{s}\right)$ for all $s \in \pi_{k}^{-1}(c)$; here $c \in\left(\mathscr{C}(k) / \Sigma_{k}\right)^{G}$. We can also think of

$$
C W \mathscr{X}=\coprod_{k} \mathscr{C}(k) \times_{\Sigma_{k}} F(\bar{k}, W \mathscr{X}) / \sim,
$$

and we could describe the subspace $C \mathscr{X}(G / G)$ in these terms. We shall use both interpretations, whichever is most convenient at the time, but it should be clear in context which one we have in mind.

We want the morphisms in $C^{G} \mathscr{X}$ to be essentially the $G$-maps between the various fibers $\pi_{k}^{-1}(c)$ and $\pi_{l}^{-1}(d)$. Precisely, to describe the space of morphisms of $C^{G} \mathscr{X}$, first consider the $G$-space

$$
M W \mathscr{X}=\coprod_{k, l} \mathscr{C}(k) \times \mathscr{C}(l) \times_{\Sigma_{k} \times \Sigma_{l}} F(\bar{l}, W \mathscr{X}) \times F(\bar{k}, \bar{l}) / \sim .
$$

Here $\Sigma_{k} \times \Sigma_{l}$ acts on $F(\bar{k}, \bar{l})$ by $((\sigma, \tau) f)(i)=f(i \sigma) \tau^{-1}$, thinking of the permutation groups as acting on the right on the sets $\bar{k}$ and $\bar{l}$. The equivalence relation is the one that has

$$
[c, d, \zeta, f] \sim\left[c^{\prime}, d^{\prime}, \zeta^{\prime}, f^{\prime}\right],
$$

if $\zeta(l)=*$ (the basepoint in $W \mathscr{X}$ ), where $\zeta^{\prime}:\{1, \ldots, l-1\} \rightarrow W \mathscr{Z}$ is the restriction of $\zeta, f^{\prime}$ is the restriction of $f$ to $\bar{k}-f^{-1}(l), d^{\prime}=\gamma(d ; 1, \ldots, 1, *)$, and $c^{\prime}=\gamma(c ; \ldots)$ where the ellipsis is filled in with a $*$ corresponding to every element of $f^{-1}(l)$, and a 1 corresponding to every element of $\bar{k}$ not in $f^{-1}(l)$. That is, as usual, things mapping to the basepoint can be thrown out. There are two $G$-maps

$$
S_{0}: M W \mathscr{X} \rightarrow C W \mathscr{X} \text { and } T: M W \mathscr{X} \rightarrow C W \mathscr{X} ;
$$

$S_{0}[c, d, \zeta, f]=[c, \zeta \circ f]$, and $T[c, d, \zeta, f]=[d, \zeta]$. The morphism space of $C^{G} \mathscr{Z}$ is then the subspace

$$
M^{G} \mathscr{X} \subset T^{-1}\left(C^{G} \mathscr{X}\right)
$$

consisting of points $[c, d, \zeta, f]$ such that $\tilde{c}=c \Sigma_{k} \in \mathscr{C}(k) / \Sigma_{k}$ and $\tilde{d}=$ $d \Sigma_{l} \in \mathscr{C}(l) / \Sigma_{l}$ are both fixed by $G$, the induced maps $\tilde{\zeta}: \pi_{l}^{-1}(\tilde{c}) \rightarrow W \mathscr{Z}$ and $\tilde{f}: \pi_{k}^{-1}(\tilde{c}) \rightarrow \pi_{l}^{-1}(\tilde{d})$ are both $G$-maps, and $\tilde{\zeta}(s) \in \mathscr{X}\left(G / G_{s}\right)$ for all $s$. The restriction of $T$ gives the target map $M^{G} \mathscr{X} \rightarrow C^{G} \mathscr{X}$. The source map $S$ is a modification of $S_{0}$ : thinking of an element in $M^{G} \mathscr{Z}$ as given by $[\tilde{c}, \tilde{d}, \tilde{\zeta}, \tilde{f}]$ as above, $S[\tilde{c}, \tilde{d}, \tilde{\zeta}, \tilde{f}]$ is the $G$-map $\xi: \pi_{k}^{-1}(c) \rightarrow W \mathscr{X}$, where 
$\xi(s)=\tilde{f}_{s}^{*} \tilde{\zeta} \tilde{f}(s), \tilde{f}_{s}: G / G_{s} \rightarrow G / G_{f(s)}$ being the restriction of $\tilde{f}$ to the orbit of $s$.

To complete the description of the topological category $C^{G} \mathscr{Z}$, we need the composition map $M^{G} \mathscr{X} \times{ }_{C^{G} \mathscr{X}} M^{G} \mathscr{X} \rightarrow M^{G} \mathscr{X}$ and the identity map $C^{G} \mathscr{X} \rightarrow$ $M^{G} \mathscr{X}$. Composition is induced by the maps

$$
F(\bar{l}, W \mathscr{X}) \times F(\bar{k}, \bar{l}) \times F(\bar{j}, \bar{k}) \rightarrow F(\bar{l}, W \mathscr{X}) \times F(\bar{j}, \bar{l}),
$$

while the identity is given by $[c, \zeta] \mapsto[c, c, \zeta, 1]$.

The last construction that we need is of a $G$-space $J^{G} \mathscr{X}$ that we shall think of as giving a continuous functor from $C^{G} \mathscr{Z}$ into $G \mathscr{T}$, assigning to each point of $C^{G} \mathscr{Z}$ the subset of the corresponding fiber $\pi_{k}^{-1}(c)$ consisting of those points not mapping to the basepoint, but with a disjoint basepoint attached. In detail, we first define

$$
J W \mathscr{X}=\coprod_{k} \mathscr{C}(k) \times \Sigma_{\Sigma_{k}} F(\bar{k}, W \mathscr{X}) \times \bar{k}^{+} / \sim,
$$

where $\bar{k}^{+}$denotes $\bar{k}$ with a disjoint basepoint $*$ added, and in addition to the usual identifications we identify $[c, \zeta, i] \sim[c, \zeta, *]$ if $\zeta(i)=*$. There is a $G$-map

\section{$T: J W \mathscr{X} \rightarrow C W \mathscr{X}$}

sending $[c, \zeta, i] \mapsto[c, \zeta]$. There is also a map $P: C W \mathscr{X} \rightarrow J W \mathscr{X}$ sending $[c, \zeta] \mapsto[c, \zeta, *]$, that we think of as giving the basepoint in each fiber of $T$. Let $J^{G} \mathscr{X}=T^{-1}\left(C^{G} \mathscr{X}\right)$, and let $T$ and $P$ also denote their restrictions to $J^{G} \mathscr{X}$ and $C^{G} \mathscr{X}$. We think of $J^{G} \mathscr{X}$ as a functor on $C^{G} \mathscr{X}$ via the evident basepoint-preserving map $M^{G} \mathscr{X} \times{ }_{C^{G} \mathscr{X}} J^{G} \mathscr{X} \rightarrow J^{G} \mathscr{X}$ covering the map $T: M^{G} \mathscr{X} \rightarrow C^{G} \mathscr{X}$. To see this as a functor $C^{G} \mathscr{X} \rightarrow G \mathscr{T}$, let $J^{G} \mathscr{X}(y)=T^{-1}(y)$ for $y \in C^{G} \mathscr{X}$.

Now we can define $E_{\mathscr{C}} \mathscr{X}$ : Let $B_{*}\left(C^{G} \mathscr{X}, C^{G} \mathscr{X}, J^{G} \mathscr{X}\right)$ be the simplicial space defined by letting $B_{n}\left(C^{G} \mathscr{X}, C^{G} \mathscr{X}, J^{G} \mathscr{X}\right)$ consist of all $(n+2)$-tuples $\left(c ; f_{1}, \ldots, f_{n} ; i\right)$, where $f_{1}, \ldots, f_{n}$ are composable maps in $C^{G} \mathscr{X}, c=$ $T\left(f_{1}\right) \in C^{G} \mathscr{Z}$, and $i \in J^{G} \mathscr{X}\left(S\left(f_{n}\right)\right)$. Actually, we make one modification to this definition, by identifying all points of the form $\left(c ; f_{1}, \ldots, f_{n} ; *\right)$. In other words,

$$
\begin{aligned}
& B_{n}\left(C^{G} \mathscr{X}, C^{G} \mathscr{X}, J^{G} \mathscr{X}\right) \\
& \quad=\left(C^{G} \mathscr{X} \times{ }_{C^{G} \mathscr{X}} M^{G} \mathscr{X} \times{ }_{C^{G} \mathscr{X}} \cdots \times{ }_{C^{G} \mathscr{X}} M^{G} \mathscr{X}\right)^{+} \wedge_{C^{G} \mathscr{X}} J^{G} \mathscr{X} .
\end{aligned}
$$

With the action of $G$ on the last factor, this makes $B_{*}\left(C^{G} \mathscr{X}, C^{G} \mathscr{X}, J^{G} \mathscr{X}\right)$ a based simplicial $G$-space, as in [M2] (see also [E]). Finally, let

$$
E_{\mathscr{C}} \mathscr{X}=B\left(C^{G} \mathscr{X}, C^{G} \mathscr{X}, J^{G} \mathscr{X}\right)
$$

be the geometric realization of this simplicial space. This construction is clearly a covariant functor of both $\mathscr{Z}$ and $\mathscr{C}$. It is a coalescence functor because we have: 
Proposition 8.1. There is a natural $\mathscr{G}$-map $\eta: \Phi E_{\mathscr{C}} \mathscr{X} \rightarrow \mathscr{X}$ which is a $\mathscr{G}$ equivalence if $\mathscr{C}$ is a complete G-operad.

Proof. $\eta$ is induced by a map $\Phi J^{G} \mathscr{X} \rightarrow \mathscr{X}$, but we shall write it out in full.

$$
\Phi E_{\mathscr{C}} \mathscr{X}(G / H)=B\left(C^{G} \mathscr{X}, C^{G} \mathscr{X},\left(J^{G} \mathscr{X}\right)^{H}\right)
$$

is the realization of $B_{*}\left(C^{G} \mathscr{X}, C^{G} \mathscr{X},\left(J^{G} \mathscr{X}\right)^{H}\right)$, which has typical element $\left(c_{0} ; f_{1}, \ldots, f_{n} ;\left[c_{n}, \zeta, t\right]\right)$. Here we think of $c_{n} \in\left(\mathscr{C}(k) / \Sigma_{k}\right)^{G}, \zeta: \pi_{k}^{-1}\left(c_{n}\right) \rightarrow$ $W \mathscr{X}$ being a $G$-map with $\zeta(s) \in \mathscr{X}\left(G / G_{s}\right)$ for all $s$, and $t \in \pi_{k}^{-1}\left(c_{n}\right)^{+}$an $H$ fixed point. $\eta$ sends this element to $p^{*} \zeta(t) \in \mathscr{X}(G / H)$, where $p: G / H \rightarrow G / G_{t}$ is induced by the inclusion $H \subset G_{t}$; if $t=*$ then $\eta$ sends this point to the basepoint of $\mathscr{X}(G / H)$. It is now easy to see that this respects the simplicial structure, and gives a $\mathscr{G}$-map, natural in $\mathscr{X}$ and $\mathscr{C}$.

To see that $\eta$ is an equivalence when $\mathscr{C}$ is complete, let $H \subset G$, and let $c \in\left(\mathscr{C}(k) / \Sigma_{k}\right)^{G}$ be a point such that $\pi_{k}^{-1}(c) \cong G / H$ as a $G$-space; let $t \in \pi_{k}^{-1}(c)$ be a point such that $G_{t}=H$. With these choices, we can define an inclusion $\mathscr{X}(G / H) \rightarrow B\left(C^{G} \mathscr{X}, C^{G} \mathscr{X},\left(J^{G} \mathscr{X}\right)^{H}\right)$ by sending $x$ to $\left(\left[c, \zeta_{x}\right] ; ;\left[c, \zeta_{x}, t\right]\right)$, where $\zeta_{x}: \pi_{k}^{-1}(c) \rightarrow W \mathscr{X}$ is the $G$-map defined by $\zeta_{x}(t)=x$. This shows that $\mathscr{X}(G / H)$ is a retract of $B\left(C^{G} \mathscr{X}, C^{G} \mathscr{X},\left(J^{G} \mathscr{X}\right)^{H}\right)$; by the usual arguments we can construct a simplicial deformation to show that $\mathscr{Z}(G / H)$ is actually a deformation retract [M1].

The point of replacing Elmendorf's construction with this one is that it makes the definition of $\lambda_{\mathscr{C}}: E_{\mathscr{C}} C \rightarrow C E_{\mathscr{C}}$ possible; this we now do. We have to define

$$
\lambda_{\mathscr{C}}: B\left(C^{G} C \mathscr{X}, C^{G} C \mathscr{X}, J^{G} C \mathscr{X}\right) \rightarrow C B\left(C^{G} \mathscr{X}, C^{G} \mathscr{X}, J^{G} \mathscr{X}\right) .
$$

This will come largely from the structure of the operad. Indeed, there is a natural functor $\mu: C^{G} C \mathscr{X} \rightarrow C^{G} \mathscr{X}$ given by that structure, being part of the map $\mu: C C \mathscr{X} \rightarrow C \mathscr{X}$. There is also a $G$-map

$$
\nu: J^{G} C \mathscr{X} \rightarrow C J^{G} \mathscr{X}
$$

defined as follows: Let $[c, \zeta, t] \in J^{G} C \mathscr{X}$, so that $c \in\left(\mathscr{C}(k) / \Sigma_{k}\right)^{G}, \zeta: \pi_{k}^{-1}(c)$ $\rightarrow W C \mathscr{X}$ with $\zeta(s) \in C \mathscr{X}\left(G / G_{s}\right)$ for all $s$, and $t \in \pi_{k}^{-1}(c)^{+}$. Let $\mu[c, \zeta]$ $=[d, \xi]$, with $d \in\left(\mathscr{C}(l) / \Sigma_{l}\right)^{G}$, and $\xi: \pi_{l}^{-1}(d) \rightarrow W \mathscr{X}$. Also, let $\zeta(t) \in$ $C \mathscr{X}\left(G / G_{t}\right)$ be represented by $[e, \varphi]$ where $e \in\left(\mathscr{C}(m) / \Sigma_{m}\right)^{G_{t}}$, and

$$
\varphi: \pi_{m}^{-1}(e) \rightarrow \bigvee_{H \subset G_{t}} \mathscr{X}(G / H)
$$

is a $G_{t}$-map. The structure of the operad gives us, for each element $u \in \pi_{m}^{-1}(e)$, an element $t * u \in \pi_{l}^{-1}(d)$. This comes from the map

$$
\mathscr{C}(k) \times \mathscr{C}\left(m_{1}\right) \times \cdots \times \mathscr{C}\left(m_{k}\right) \times\{1\} \times \bar{m}_{1} \rightarrow \mathscr{C}(l) \times \bar{l}
$$


given by $\left(c ; e_{1}, \ldots, e_{m} ; 1, u\right) \mapsto\left(\gamma\left(c ; e_{i}\right), u\right)$; here we represent $t$ by $1 \in \bar{k}$, and think of $m=m_{1}$, while $l=m_{1}+\cdots+m_{k}$. Now we let $\nu[c, \zeta, t]=[e, \psi]$ where $\psi: \pi_{m}^{-1}(e) \rightarrow J^{G} \mathscr{X}$ is defined by $\psi(u)=[d, \xi, t * u]=[\mu[c, \zeta], t * u]$.

Finally, we define

$$
\lambda_{\mathscr{C}}\left(c_{0} ; f_{1}, \ldots, f_{n} ;\left[c_{n}, \zeta, t\right]\right)=\left[e, \psi^{\prime}\right],
$$

where $\nu\left[c_{n}, \zeta, t\right]=[e, \psi]$ as above, and

$$
\begin{aligned}
\psi^{\prime}(u) & =\left(\mu\left(c_{0}\right) ; \mu\left(f_{1}\right), \ldots, \mu\left(f_{n}\right) ; \psi(u)\right) \\
& =\left(\mu\left(c_{0}\right) ; \mu\left(f_{1}\right), \ldots, \mu\left(f_{n}\right) ;\left[\mu\left[c_{n}, \zeta\right], t * u\right]\right) .
\end{aligned}
$$

That this defines a natural $G$-map is now straightforward to check. Since $\lambda_{\mathscr{C}}$ is defined using the operad structure, it is also simple to check that the diagrams of Proposition 4.2 commute. Finally, we show:

Proposition 8.2. If $\mathscr{C}$ is complete, then $\lambda_{\mathscr{C}}$ is a G-equivalence.

Proof. First notice that the diagram

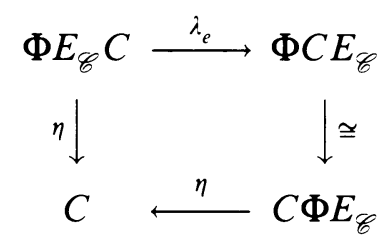

commutes, as can be checked from the definitions of the maps involved. However, we have already shown that $\eta$ is an equivalence, therefore $\lambda_{\mathscr{C}}$ must be an equivalence.

\section{REFERENCES}

[CW] J. Caruso and S. Waner, An approximation theorem for equivariant loop spaces in the compact Lie case, Pacific J. Math. 117 (1985), 27-49.

[CHMW] S. R. Costenoble, H. Hauschild, J. P. May, and S. Waner, Equivariant infinite loop space theory (to appear).

[D1] A. Dress, Contributions to the theory of induced representations, Lecture Notes in Math., vol. 342, Springer-Verlag, 1973, pp. 183-240.

[D2] _ Induction and structure theorems for orthogonal representations of finite groups, Ann. of Math. 102 (1975), 291-325.

[E] A. D. Elmendorf, Systems of fixed point sets, Trans. Amer. Math. Soc. 277 (1983), 275-284.

[HW] H. Hauschild and S. Waner, The equivariant Dold theorem $\bmod k$ and the Adams conjecture, Illinois J. Math. 27 (1983), 52-66.

[LMM] G. Lewis, Jr., J. P. May, and J. McClure, Ordinary equivariant $R O(G)$-graded cohomology, Bull. Amer. Math. Soc. 4 (1981), 208-212.

[LMS] G. Lewis, Jr., J. P. May, and M. Steinberger, with contributions by J. E. McClure, Equivariant stable homotopy theory, Lecture Notes in Math., vol. 1213, Springer-Verlag, 1986.

[M1] J. P. May, The geometry of iterated loop spaces, Lecture Notes in Math., vol. 271, SpringerVerlag, 1972.

[M2] _ Classifying spaces and fibrations, Mem. Amer. Math. Soc., no. 155 (1972).

[M3],$- E_{\infty}$ ring spaces and $E_{\infty}$ ring spectra, Lecture Notes in Math., vol. 577, SpringerVerlag, 1977. 
[S] G. B. Segal, Configuration spaces and iterated loop spaces, Invent. Math. 21 (1973), 213221.

[W1] S. Waner, Equivariant homotopy theory and Milnor's Theorem, Trans. Amer. Math. Soc. 258 (1980), 351-368.

[W2] _ Equivariant classifying spaces and fibrations, Trans. Amer. Math. Soc. 258 (1980), 385-405.

[W3] _ Three topological categories of G-spaces, Hofstra University (preprint).

Department of Mathematics, Hofstra University, Hempstead, New York 11550 